\section{MS43-P12 Also disorder can improve thin film transistor performance}

Laura Ferlauto ${ }^{1}$, Fabiola Liscio $^{1}$, Emanuele Orgiu ${ }^{2}$, Norberto Masciocchi $^{3}$, Antonietta Guagliardi ${ }^{4}$, Fabio Biscarini ${ }^{5}$, Paolo Samorì ${ }^{2}$, Silvia Milita

1. Istituto di Microelettronica e Microsistemi (IMM)-CNR, via Piero Gobetti 101, I-40129, Bologna, Italy

2. ISIS \& icFRC, Université de Strasbourg \& CNRS, 8 allée Gaspard Monge 67000, Strasbourg, France

3. Dip. Scienza e Alta Tecnologia, Università dell'Insubria \& To.Sca.Lab, via Valleggio 11, I-22100 Como, Italy

4. Istituto di Cristallografia-CNR \& To.Sca.Lab, via Lucini 3, I-22100 Como, Italy

5. Dip. Scienze della Vita, Università di Modena e Reggio Emilia, via Campi 183, 41125, Modena, Italy

email: ferlauto@bo.imm.cnr.it

The relentless progress in Organic Electronics has catalyzed the attention to the design of novel materials combining structural and functional complexity. Despite this, the development of highly performing solution processed n-type Organic Field-Effect Transistors is still a great challenge due to the difficulty of achieving high solubility and good air stability without undermining electrical properties. Among n-type organic semiconductors, perylene alkyldiimide derivatives have demonstrated good electrical performance as well as excellent processability and air stability. In particular, the functionalization of the perylene core by means of cyano-groups increases the ambient stability by lowering the energy of the lowest unoccupied molecular orbital, while the presence of alkyl chains enhances the solubility of the molecules by decreasing the core planarity. Since the introduction of side chains also affects the self-assembly at the supramolecular level and consequently also the efficiency of charge transport in the material, a cautious design which aims to optimize the intermolecular interactions between $\pi$-conjugated molecules must be implemented. This study emphasizes how the introduction of molecular disorder can be the key for enhanced transport properties once the devices are treated via thermal annealing. In this work we report a multiscale structural analysis performed by X-ray diffraction, X-ray reflectivity and atomic force microscopy on thin-films of dicyanoperylene molecules decorated with either linear or asymmetric branched alkyl-side chains. The synthesis of the branched species leads to the formation of a mixture of four "distinct" stereoisomers, two RR/SS and RS/SR enantiomeric pairs. This conformational disorder not only increases the material solubility and makes the crystallization process more difficult with respect to the case of molecules with linear side-chains, but also favors the 2D-growth mode. Optimized post-deposition thermal annealing leads then to a structural transition towards the bulk-phase for molecules exposing branched chains, still preserving the 2D morphology and improving the transport properties of thin-film devices. These findings suggest that the synthesis of highly processable molecules capable of undergoing strong supramolecular rearrangement during optimized classical post-growth processes can be more efficient than improving the charge carrier mobility at the expense of solubility.

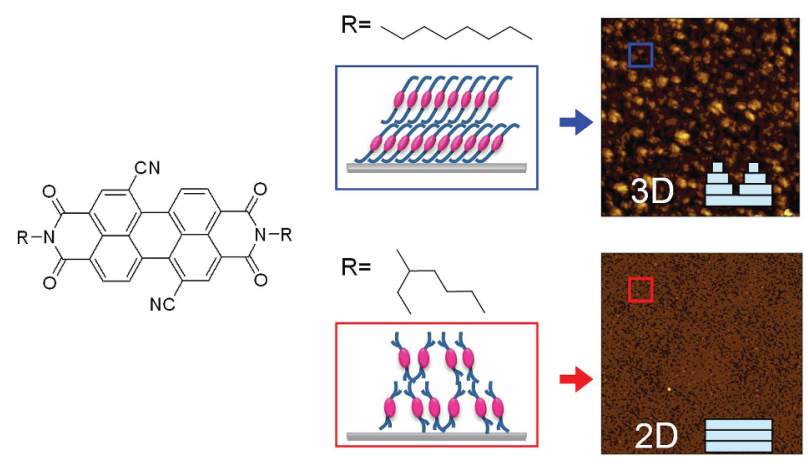

Figure 1. Chemical formula, sketch of molecular self-assembly and AFM images of the two perylene di-imide isomers.

Keywords: organic field-effect transistors, organic thin films, $\mathrm{X}$-ray diffraction, perylene derivatives 